University of Wollongong

Research Online

Faculty of Engineering - Papers (Archive)

Faculty of Engineering and Information

Sciences

$1-1-2007$

\title{
Space-time-frequency codes in MB-OFDM UWB communications: advanced order-8 STFC and its performance
}

\author{
Le Chung Tran \\ University of Lubeck, Ictran@uow.edu.au
}

\author{
A. Mertins \\ University of Wollongong, mertins@uow.edu.au \\ Eryk Dutkiewicz \\ University of Wollongong, eryk@uow.edu.au \\ Xiaojing Huang \\ University of Wollongong, huang@uow.edu.au
}

Follow this and additional works at: https://ro.uow.edu.au/engpapers

Part of the Engineering Commons

https://ro.uow.edu.au/engpapers/4246

\section{Recommended Citation}

Tran, Le Chung; Mertins, A.; Dutkiewicz, Eryk; and Huang, Xiaojing: Space-time-frequency codes in MBOFDM UWB communications: advanced order-8 STFC and its performance 2007, 380-385.

https://ro.uow.edu.au/engpapers/4246

Research Online is the open access institutional repository for the University of Wollongong. For further information contact the UOW Library: research-pubs@uow.edu.au 


\section{Space-Time-Frequency Codes in MB-OFDM UWB Communications: Advanced Order- 8 STFC and its Performance}

\author{
L. C. Tran, A. Mertins \\ Institute for Signal Processing \\ University of Lübeck, Germany \\ Email: \{tran,mertins\}@isip.uni-luebeck.de
}

\author{
E. Dutkiewicz, and X. Huang \\ School of Elec. Comp. \& Telecomm. Eng. \\ University of Wollongong, Australia \\ Email: \{eryk,huang\}@uow.edu.au
}

\begin{abstract}
The paper examines the potential of the implementation of high-order Space-Time-Frequency Codes (STFCs) in recently proposed STFC Multi-Band OFDM Ultra Wideband (MB-OFDM UWB) communications systems, and compares the performance of two different code structures of order-8 to that of the Alamouti code and of the conventional MB-OFDM UWB system (without STFCs). It is shown that an order-8 STFC provides significantly better error performance, compared to the Alamouti STFC, and much better error performance, compared to the conventional MB-OFDM, without increasing the total transmission power. It is also shown that, for the same total transmission power, the order-8, fully structured code that we proposed previously for conventional wireless Multiple-Input Multiple-Output (MIMO) Space-Time Block Codes (STBCs) communications is an advanced code, providing the best error performance.
\end{abstract}

\section{INTRODUCTION}

Multiband Orthogonal Frequency Division Multiplexing U1tra Wideband (MB-OFDM UWB) technology [1] is a potential candidate for short range communications (up to 10 meters) with a very high rate (480 Mbps), low power consumption and emission, and at a low cost. On the other hand, Multiple Input Multiple Output (MIMO) systems using multiple transmit (Tx) antennas and/or multiple receive $(\mathrm{Rx})$ antennas have recently been intensively examined, because they can potentially provide a very high system capacity, which in various cases grows linearly with the maximum number of $\mathrm{Tx}$ and $\mathrm{Rx}$ antennas, without any increase of the total transmission power [2], [3]. Space-Time Codes (STCs) [4], [5], [6], [7], [8] are the codes designed for the use of MIMO systems. Intuitively, the combination between the three emerging techniques MBOFDM UWB, MIMO, and STCs is a promising candidate for significantly improving the performance of the conventional MB-OFDM UWB system and for satisfying the needs of multimedia communications between portable devices with very high data rates, improved communications quality, and low cost.

However, this combination has been almost unexplored in the literature, with few published papers, such as [9], [10], [11], [12]. In particular, the combination between MB-OFDM UWB systems with Space-Time Block Codes (STBCs) has been somewhat mentioned in [9] for only $2 \mathrm{Tx}$ antennas, ie. the Alamouti code [4]. In [10], the authors proposed a general framework to analyze the performance of MBOFDM MIMO UWB systems regardless of specific coding schemes. They quantify the performance of the MB-OFDM MIMO UWB systems in case of Nakagami frequency-selective fading channels. The authors also showed that the maximum achievable diversity of a MIMO MB-OFDM UWB system is the product of the number of Tx and Rx antennas, the number of multipath components, and the number of jointly encoded OFDM symbols.

Unfortunately, the multipath channel amplitudes in MBOFDM UWB systems are independent log-normally distributed random variables, rather than Nakagami-distributed ones [13]. While [10] is a very interesting paper and has significant contribution, it still has some drawbacks, including: a) not quantifying the Pairwise Error Probability (PEP) in the case of log-normal distribution, b) assuming that the time delay and average power are the same for all transmit-receive links in order to calculate the matrix of channel frequency responses, which is not usually true in MB-OFDM systems where the time delay and average power of transmit-receive links might be very different, and c) misjudging that the product between the channel's multipath length and the number of Tx antennas is usually smaller than the FFT size, resulting in an erroneous conclusion that the maximum achievable diversity of MBOFDM UWB systems is the product of the number of Tx and $\mathrm{Rx}$ antennas, the number of multipath components, and the number of jointly encoded OFDM symbols. In fact, the product between the channel's multipath length and the number of Tx antennas is usually much greater than the FFT size, due to the fact that UWB channels are very richly dispersive.

In [14], we expanded the discussion in [9] and proposed STFC MB-OFDM UWB systems for any number of Tx/Rx antennas. We followed an approach for examining the performance of STFC MB-OFDM UWB systems that is independent from the existing works. In particular, we modified Tarokh's proof, which was mentioned in [5] for the conventional wireless STC MIMO communications, to quantify the diversity and coding gains of the proposed STFC MB-OFDM UWB system in the log-normal distribution case [15], without any restriction or additional assumption of the time delay and average power of transmit-receive links. Our analysis was based closely on WiMedia's MB-OFDM UWB PHY specifications [1] and the IEEE 802.15.3a UWB channel model [13]. We discovered that the maximum achievable diversity gain of the proposed STFC MB-OFDM UWB system is the product of the numbers of Tx and Rx antennas and the FFT size. 


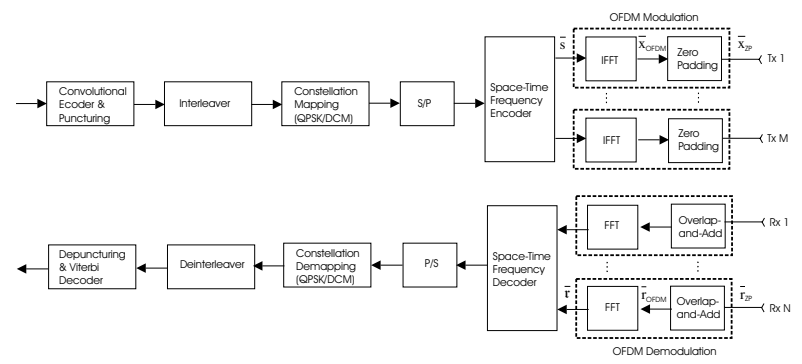

Fig. 1. Structural diagram of the proposed STFC MB-OFDM UWB systems.

In this paper, we analyze the advantage of high-order STFCs and the feasibility of their deployment. Though the full rate cannot be achieved by high-order STFCs, a higher diversity order can be achieved. Thus, the error performance of STFC MB-OFDM can be significantly improved, with the cost of reduced data rates. We also point out that the fully-structured codes comprising no zero element, which we have proposed previously for wireless MIMO STBC systems [7], [16], are advanced structures, providing better error performance than the conventional code comprising various zeros, without any increase of the total transmission power.

The paper is organized as follows. In Section II, we review the specifications of the STFC MB-OFDM system proposed in [14]. Section III analyzes the feasibility of the deployment of up to 8th order STFCs in the STFC MB-OFDM system, and analyzes the advantages of our advanced STFC structure. Section IV shows various simulation results to verify our analysis. Conclusions are drawn in Section V.

\section{STFC MB-OFDM UWB SYSTEM}

The diagram of the proposed STFC MB-OFDM UWB system with the notations of signals at the considered reference points is depicted in Fig. 1. The system consists of $M$ Tx antennas and $N \mathrm{Rx}$ antennas. We assume that the transmitted STFC is the following matrix

$$
\mathbf{S}=\left\{\overline{\mathbf{s}}_{t, m}\right\}_{T \times M} .
$$

Structures of $\mathbf{S}$ are the same as those of Complex Orthogonal STBCs (CO STBCs) in conventional wireless STBC MIMO systems [4], [7], [16], [17], [6], except that each element $\overline{\mathbf{s}}_{t, m}$ is not a complex number, but defined as a column vector $\overline{\mathbf{s}}_{t, m}=\left[s_{t, m, 1}, s_{t, m, 2}, \ldots, s_{t, m, N_{f f t}}\right]^{T}$. The vectors $\overline{\mathbf{s}}_{t, m}$ are the original transmitted data before IFFT. The symbols $s_{t, m, k}$ are drawn from a QPSK or Dual Carrier Modulation (DCM) constellation. The superscript ${ }^{T}$ denotes the transposition operation.

Elements $\overline{\mathbf{s}}_{t, m}$ in each row of $\mathbf{S}$ are transmitted simultaneously through $M$ Tx antennas in the same frequency band, while different rows of $\mathbf{S}$ might be transmitted in different frequency bands, following a certain Time-Frequency Code (TFC). Different TFCs (transmitted RF patterns) are described in more details in [1].

Denote $\mathcal{X}=\left\{\overline{\mathbf{x}}_{O F D M, t, m}\right\}_{T \times M}$ to be the matrix whose elements are the $N_{f f t}$-point IFFTs of the respective elements in $\mathbf{S}$, then

$$
\begin{aligned}
\mathcal{X} & =\left\{\operatorname{IFFT}\left\{\overline{\mathbf{s}}_{t, m}\right\}\right\}_{T \times M} \\
& =\left\{\overline{\mathbf{x}}_{O F D M, t, m}\right\}_{T \times M} .
\end{aligned}
$$

TABLE I

Numbers of Multipaths $N p_{10 d B}, N p_{85 \%}$, AND $\bar{N}_{p}$ [13].

\begin{tabular}{l|r|r|r|r}
\hline & CM 1 & CM 2 & CM 3 & CM 4 \\
\hline$N p_{10 d B}$ & 12.5 & 15.3 & 24.9 & 41.2 \\
$N p_{85 \%}$ & 20.8 & 33.9 & 64.7 & 123.3 \\
$\bar{N}_{p}$ & 287.9 & 739.5 & 1463.7 & 3905.5 \\
\hline
\end{tabular}

The symbols $\overline{\mathbf{x}}_{O F D M, t, m}$ are referred to as MB-OFDM symbols. Further, denote $\mathcal{X}_{\mathbf{Z P}}=\left\{\overline{\mathbf{x}}_{Z P t, m}\right\}_{T \times M}$ to be the matrix whose elements are the respective elements in $\mathcal{X}$ appended by a Zero-Padded Suffix (ZPS) of 37 zeros. Clearly, $\overline{\mathbf{x}}_{O F D M, t, m}$ is the transmitted MB-OFDM symbol before Zero Padding (ZP), while $\overline{\mathbf{x}}_{Z P, t, m}$ is the actual transmitted symbol after ZP. Denote

$$
\overline{\mathbf{h}}_{m, n}=\left[\begin{array}{llll}
h_{m, n, 1} & h_{m, n, 2} & \ldots h & m, n, L_{m, n}
\end{array}\right]^{T}
$$

to be the channel vector between the $m$-th $\mathrm{Tx}$ and $n$-th $\mathrm{Rx}$ antennas, for $m=1, \ldots, n=1, \ldots, N$, where the channel coefficients $h_{m, n, l}$ of the $l$-th path, $l=1, \ldots, L_{m, n}$, in this channel are modeled as independent log-normally distributed random variables (RVs). Let $L_{\max }=\max \left\{L_{m, n}\right\}$, for $m=1, M h, \quad=1, \ldots, N$. Denote the MB-OFDM UWB channel coefficient matrix as

$$
\mathbf{H}=\left\{\overline{\mathbf{h}}_{m, n, Z P}\right\}_{M \times N}
$$

where the vector $\overline{\mathbf{h}}_{m, n, Z P}$ is created from the corresponding channel vector $\overline{\mathbf{h}}_{m, n}$ by adding zeros to have the length $L_{\text {max }}$.

At the transmission of the $t$-th MB-OFDM symbol, the received signal at the $n$-th $\mathrm{Rx}$ antenna is calculated as

$$
\overline{\mathbf{r}}_{Z P, t, n}=\sum_{m=1}^{M}\left(\overline{\mathbf{x}}_{Z P, t, m} \otimes \overline{\mathbf{h}}_{m, n}\right)+\overline{\mathbf{n}}_{t, n}
$$

where $\otimes$ denotes the linear convolution. The elements of the noise vector $\overline{\mathbf{n}}_{t, n}$ are considered to be independent complex Gaussian RVs.

\section{A. Theoretical Analysis}

In this section, we first analyze the proposed system with the theoretical assumption that the maximum number of multipaths $L_{m, n}$, for $m=1,, M n=1, N$., , is $\left(N_{Z P S}+1\right)$, where $N_{Z P S}$ denotes the length of the ZPS. Instead of inserting a $\mathrm{CP}$ at the transmitter and discarding the $\mathrm{CP}$ at the receiver as in a conventional OFDM system, in MB-OFDM system, a ZPS of a length $N_{Z P S}$ is appended to each symbol $\overline{\mathbf{x}}_{O F D M, t, m}$ at the transmitter to create a transmitted symbol $\overline{\mathbf{x}}_{Z P, t, m}$. At the receiver, an OverlapAnd-Add Operation (OAAO) must be performed before FFT. OAAO means that the $N_{Z P S}$ samples of a received symbol $\overline{\mathbf{r}}_{Z P, t, n}$, ranging from $\left(N_{f f t}+1\right)$ to $\left(N_{f f t}+N_{Z P S}\right)$, are added to the beginning of that received symbol. Then the first $N_{f f t}$ samples of the resulting symbol will be used to decode the transmitted symbol. These $N_{f f}$ samples are exactly equivalent to the circular convolution of the transmitted OFDM symbol (before ZP) $\overline{\mathbf{x}}_{O F D M, t, m}$ with the channel $\overline{\mathbf{h}}_{m, n}$. This exact equivalence is due to the fact that, if a ZPS of a length $N_{Z P S}$ is used, the greatest multipath tolerance of the system is $\left(N_{Z P S}+1\right)$. Consequently, from the theoretical viewpoint, the number of multipaths (the length of vectors $\overline{\mathbf{h}}_{m, n}$ ) must not exceed $\left(N_{Z P S}+1\right)$. 
As a result, after performing OAAO for the received signal $\overline{\mathbf{r}}_{Z P, t, n}$ in (6) and then taking the first $N_{f f t}$ resulting samples, denoted as $\overline{\mathbf{r}}_{O F D M, t, n}$, the following equation is deduced

$$
\overline{\mathbf{r}}_{O F D M, t, n}=\sum_{m=1}^{M} \overline{\mathbf{x}}_{O F D M, t, m} * \overline{\mathbf{h}}_{m, n}+\overline{\mathbf{n}}_{t, n}
$$

where $*$ denotes the cyclic convolution or circular convolution. For the circular convolution, we have the following property

$$
\begin{aligned}
\overline{\mathbf{x}}_{O F D M, t, m} * \overline{\mathbf{h}}_{m, n}= & \operatorname{IFFT}\left\{F F \left\{\overline{\mathbf{x}}_{O F D M, t, m} \bullet \bullet\right.\right. \\
& \left.F F T\left\{\overline{\mathbf{h}}_{m, n}\right\}\right\} \\
= & \operatorname{IFFT}\left\{\overline{\mathbf{s}}_{t, m} \bullet \overline{\mathfrak{h}}_{m, n}\right\}
\end{aligned}
$$

where $\bullet$ denotes the element-wise or Hadamard product, and $\overline{\mathfrak{h}}_{m, n}$ is the $N_{f f t}$-point FFT of the channel vector $\overline{\mathbf{h}}_{m, n}$, ie.

$$
\overline{\mathfrak{h}}_{m, n}=F F T\left\{\overline{\mathbf{h}}_{m, n}\right\}
$$

We denote $\overline{\mathfrak{h}}_{m, n}=\left[\begin{array}{llll}\hbar_{m, n, 1} & \hbar_{m, n, 2} & \ldots & \hbar_{m, n, N_{f f t}}\end{array}\right]^{T}$. After going through the FFT block at the receiver, the received signal becomes

$$
F F T\left\{\overline{\mathbf{r}}_{O F D M, t, n}\right\}=\sum_{m=1}^{M} \overline{\mathbf{s}}_{t, m} \bullet \overline{\mathfrak{h}}_{m, n}+F F T\left\{\overline{\mathbf{n}}_{t, n}\right\}
$$

$$
\begin{aligned}
& \text { Denote } \\
& \quad \overline{\mathfrak{r}}_{t, n}=\left[\begin{array}{llll}
\mathfrak{r}_{t, n, 1} & \mathfrak{r}_{t, n, 2} & \ldots & \mathfrak{r}_{t, n, N_{f f t}}
\end{array}\right]^{T}=F F T\left\{\overline{\mathbf{r}}_{t, n}\right\} \\
& \text { and } \\
& \quad \overline{\mathfrak{n}}_{t, n}=\left[\begin{array}{llll}
\mathfrak{n}_{t, n, 1} & \mathfrak{n}_{t, n, 2} & \ldots & \mathfrak{n}_{t, n, N_{f f t}}
\end{array}\right]^{T}=\operatorname{FFT}\left\{\overline{\mathbf{n}}_{t, n}\right\} .
\end{aligned}
$$

Then (10) can be rewritten as follows

$$
\overline{\mathfrak{r}}_{t, n}=\sum_{m=1}^{M} \overline{\mathbf{s}}_{t, m} \bullet \overline{\mathfrak{h}}_{m, n}+\overline{\mathfrak{n}}_{t, n} .
$$

Recall that $\overline{\mathbf{s}}_{t, n}$ is the original QPSK or DCM transmitted signal (before IFFT).

Denote $\mathcal{H}=\left\{\overrightarrow{\mathfrak{h}}_{m, n}\right\}_{M \times N}$ to be the matrix whose elements are the $N_{f f t}$-point FFTs of the respective elements in the channel coefficient matrix $\mathbf{H}$. Further, denote $\mathbf{R}=$ $\left\{\overline{\mathbf{r}}_{O F D M, t, n}\right\}_{T \times N}$ to be the received signal matrix, $\mathcal{R}=$ $\left\{\overline{\mathfrak{r}}_{t, n}\right\}_{T \times N}$ to be the received signal matrix after FFT, and $\mathcal{N}=\left\{\overline{\mathfrak{n}}_{t, n}\right\}_{T \times N}$ to be noise matrix.

We can rewrite (11) in matrix form as follows

$$
\mathcal{R}=\mathrm{S} \circ \mathcal{H}+\mathcal{N}
$$

where we define the multiplication operation $\circ$ between $\mathbf{S}$ and $\mathcal{H}$ as that the $(t, n)$-th element of the resulting matrix is a $N_{f \boldsymbol{t}}$-length column vector $\sum_{m=1}^{M} \overline{\mathbf{s}}_{t, m} \bullet \overline{\mathfrak{h}}_{m, n}$.

From (12), we can realize that there exists a similarity between the mathematical model of the STFC MB-OFDM UWB system and that of the conventional wireless STC MIMO system [4], [7], [18]. The only difference between the two mathematical models is that elements in each matrix are numbers in the conventional STC MIMO system, while they are $N_{f f}$-length column vectors in the STFC MB-OFDM UWB system.

Because the vector elements in $\mathbf{S}$ will be transformed with the IFFT to generate MB-OFDM symbols with $N_{f f t}$ subcarriers, we refer to $\mathbf{S}$ as a Space-Time-Frequency Code.

\section{B. Realistic Channel Conditions}

The error performance of the proposed system with realistic UWB channel conditions is inferior, compared to the theoretical performance, due to the two following main reasons.

In theory, the length of the CP or ZPS must be greater than the longest multipath in an OFDM-based system to turn the linear convolution between the transmitted signal and the channel vector into the circular convolution. However, in practice, the multipath length is very likely to exceed the length of CP or ZPS. It is especially true in MB-OFDM UWB systems where the average number of multipaths $\bar{N}_{p}$ is usually much bigger than $N_{Z P S}=37$ (see Table I). The transition from (6) to (11) is an approximation, due to the fact that the circular convolution in (8) is approximate, but not exactly equal to the first $N_{f f t}$ samples achieved by the OAAO of the linear convolution $\overline{\mathbf{x}}_{Z P, t, m} \otimes \overline{\mathbf{h}}_{m, n}$ in (6). The energy of multipath components within the ZPS window will be captured, while the multipath components outside this window may be considered as interferences for the received signals. Eq. (6) represents the real received signals at the $\mathrm{Rx}$ antennas, while (11) shows the realistic concept used at the STFC decoder to decode the original transmitted signals. By calculating the received signals $\overline{\mathbf{r}}_{Z P, t, n}$ with the full impact of long multipath channels (with the linear convolution) based on (6), and decoding signals based on (11), ie. based on the circular convolution, we can simulate the performance of the proposed system, which is close to the realistic performance of the system. Thereby, we can find out how the multipath channels actually impact on the system performance.

On the other hand, for a channel vector $\overline{\mathbf{h}}_{m, n}=$ $\left[h_{m, n, 1}, h_{m, n, 2}, \ldots h{ }_{m, n, L_{m, n}}\right]^{T}$, we always have the following property for the $N_{f f t}$-point FFT operation

$$
\operatorname{FFT}\left\{\overline{\mathbf{h}}_{m, n}\right\}=\operatorname{FFT}\left\{\left[\begin{array}{llll}
h_{m, n, 1} & \ldots & h & m, n, N_{f f t}
\end{array}\right]^{T}\right\}
$$

if the length $L_{m, n}$ of the vector is not smaller than $N_{f f t}$. This means that, by transforming the received signals with a limited FFT size $N_{f t \boldsymbol{t}}$ and decoding signals based on (11), the $N_{f f t}$-point FFT operation truncates the impact of a long vector $\mathbf{h}_{m, n}$ to the length of $N_{f f t}$. Therefore, the higher $N_{f f t}$ is, the closer the approximation between the linear convolution and the circular convolution is, and thus the better the system performance is. However, FFT and IFFT blocks significantly determine the complexity and the cost of transmitter and receiver. As a result, there must be a suitable compromise between the cost/complexity and the system performance.

\section{Advanced Order-8 STFC ANd ItS Performance}

If the code rate of an STFC is defined as the ratio of the number of transmitted MB-OFDM symbols and the number of time slots required to transmit the whole block of the code, it is well known that the Alamouti code in (14) can provide a full rate for two Tx antennas

$$
\mathbf{S}_{\mathbf{2}}=\left[\begin{array}{rr}
\overline{\mathbf{s}}_{1} & \overline{\mathbf{s}}_{2} \\
-\overline{\mathbf{s}}_{2}^{*} & \overline{\mathbf{s}}_{1}^{*}
\end{array}\right]
$$

while higher-order codes for more than two Tx antennas cannot provide the full rate. 
However, they can still provide a full diversity order for those number of Tx antennas. As a result, the higher-order codes can provide better error performance without any increase of the total transmission power, with the penalty of lower code rates, compared to the Alamouti code. Therefore, the implementation of higher-order STFCs for multiple Tx/Rx antennas in STFC MB-OFDM UWB communications is still of strong interest.

In order to avoid the spatial correlation between Tx and/or $\mathrm{Rx}$ antennas, the antennas should be located by multiples of $\lambda / 2$, where $\lambda$ is the wavelength, apart from each other. Let us consider the case of order- 8 STFCs ( 8 Tx antennas are required) for instance. We assume that the Tx antennas are separated from one another by $\lambda / 2$, which is in the range of 14.2-48.4 mm for the UWB frequency range 3.1-10.6 GHz, then the length of UWB devices locating 8 Tx antennas should be about $9.9-33.9 \mathrm{~cm}$. Clearly, this length is the typical length of wireless devices, such as wireless access points or routers. Thus it can be stated that the application of up to $8 \mathrm{Tx}$ antennas can be feasible in STFC MB-OFDM UWB communications, because devices work at a very high center frequency.

Various constructions of order-8 Complex Orthogonal STBCs (CO STBCs) have been proposed in the literature for conventional wireless MIMO STBC systems, including codes with various zeros inside the code matrix [8], [19], and those advanced codes without any zero [7], [16]. Examples of the STFCs based on the conventional structures with various zeros [19], and based on our advanced codes [7] are derived in (15) and (16), respectively.

$\mathbf{S}_{\mathbf{8 a}}=\left[\begin{array}{rrrrrrrr}\overline{\mathbf{s}}_{1} & -\overline{\mathbf{s}}_{2}^{*} & -\overline{\mathbf{s}}_{3}^{*} & 0 & -\overline{\mathbf{s}}_{4}^{*} & 0 & 0 & 0 \\ \overline{\mathbf{s}}_{2} & \overline{\mathbf{s}}_{1}^{*} & 0 & \overline{\mathbf{s}}_{3}^{*} & 0 & \overline{\mathbf{s}}_{4}^{*} & 0 & 0 \\ \overline{\mathbf{s}}_{3} & 0 & \overline{\mathbf{s}}_{1}^{*} & -\overline{\mathbf{s}}_{2}^{*} & 0 & 0 & \overline{\mathbf{s}}_{4}^{*} & 0 \\ 0 & -\overline{\mathbf{s}}_{3} & \overline{\mathbf{s}}_{2} & \overline{\mathbf{s}}_{1} & 0 & 0 & 0 & -\overline{\mathbf{s}}_{4}^{*} \\ \overline{\mathbf{s}}_{4} & 0 & 0 & 0 & \overline{\mathbf{s}}_{1}^{*} & -\overline{\mathbf{s}}_{2}^{*} & -\overline{\mathbf{s}}_{3}^{*} & 0 \\ 0 & -\overline{\mathbf{s}}_{4} & 0 & 0 & \overline{\mathbf{s}}_{2} & \overline{\mathbf{s}}_{1} & 0 & \overline{\mathbf{s}}_{3}^{*} \\ 0 & 0 & -\overline{\mathbf{s}}_{4} & 0 & \overline{\mathbf{s}}_{3} & 0 & \overline{\mathbf{s}}_{1} & -\overline{\mathbf{s}}_{2}^{*} \\ 0 & 0 & 0 & \overline{\mathbf{s}}_{4} & 0 & -\overline{\mathbf{s}}_{3} & \overline{\mathbf{s}}_{2} & \overline{\mathbf{s}}_{1}^{*}\end{array}\right]$

$\mathbf{S}_{\mathbf{8 b}} \quad=\left[\begin{array}{rrrrrrrr}\overline{\mathbf{s}}_{1} & \overline{\mathbf{s}}_{1} & -\overline{\mathbf{s}}_{2}^{*} & \overline{\mathbf{s}}_{2}^{*} & -\overline{\mathbf{s}}_{3}^{*} & \overline{\mathbf{s}}_{3}^{*} & -\overline{\mathbf{s}}_{4}^{*} & \overline{\mathbf{s}}_{4}^{*} \\ -\overline{\mathbf{s}}_{1}^{*} & \overline{\mathbf{s}}_{1}^{*} & \overline{\mathbf{s}}_{2} & \overline{\mathbf{s}}_{2} & \overline{\mathbf{s}}_{3} & \overline{\mathbf{s}}_{3} & \overline{\mathbf{s}}_{4} & \overline{\mathbf{s}}_{4} \\ \overline{\mathbf{s}}_{2}^{*} & -\overline{\mathbf{s}}_{2}^{*} & \overline{\mathbf{s}}_{1} & \overline{\mathbf{s}}_{1} & -\overline{\mathbf{s}}_{4} & \overline{\mathbf{s}}_{4} & \overline{\mathbf{s}}_{3} & -\overline{\mathbf{s}}_{3} \\ -\overline{\mathbf{s}}_{2} & -\overline{\mathbf{s}}_{2} & -\overline{\mathbf{s}}_{1}^{*} & \overline{\mathbf{s}}_{1}^{*} & \overline{\mathbf{s}}_{4}^{*} & \overline{\mathbf{s}}_{4}^{*} & -\overline{\mathbf{s}}_{3}^{*} & -\overline{\mathbf{s}}_{3}^{*} \\ \overline{\mathbf{s}}_{3}^{*} & -\overline{\mathbf{s}}_{3}^{*} & \overline{\mathbf{s}}_{4} & -\overline{\mathbf{s}}_{4} & \overline{\mathbf{s}}_{1} & \overline{\mathbf{s}}_{1} & -\overline{\mathbf{s}}_{2} & \overline{\mathbf{s}}_{2} \\ -\overline{\mathbf{s}}_{3} & -\overline{\mathbf{s}}_{3} & -\overline{\mathbf{s}}_{4}^{*} & -\overline{\mathbf{s}}_{4}^{*} & -\overline{\mathbf{s}}_{1}^{*} & \overline{\mathbf{s}}_{1}^{*} & \overline{\mathbf{s}}_{2}^{*} & \overline{\mathbf{s}}_{2}^{*} \\ \overline{\mathbf{s}}_{4}^{*} & -\overline{\mathbf{s}}_{4}^{*} & -\overline{\mathbf{s}}_{3} & \overline{\mathbf{s}}_{3} & \overline{\mathbf{s}}_{2} & -\overline{\mathbf{s}}_{2} & \overline{\mathbf{s}}_{1} & \overline{\mathbf{s}}_{1} \\ -\overline{\mathbf{s}}_{4} & -\overline{\mathbf{s}}_{4} & \overline{\mathbf{s}}_{3}^{*} & \overline{\mathbf{s}}_{3}^{*} & -\overline{\mathbf{s}}_{2}^{*} & -\overline{\mathbf{s}}_{2}^{*} & -\overline{\mathbf{s}}_{1}^{*} & \overline{\mathbf{s}}_{1}^{*}\end{array}\right]$

The advanced codes have the following advantages

1) Unlike in conventional codes with various zero elements, it is not required either to turn off the Tx antennas during transmission, or to waste transmit power. Turning off Tx antennas during transmission is inconvenient, especially for a very high data rate system, while transmitting no useful information wastes the system resources.

2) A lower average signal-constellation energy (SCE) can be used to achieve the same bit error performance as for the conventional codes with zeros. Equivalently, with the same SCE, the advanced codes provide better error performance, compared to the conventional codes.
TABLE II

SimUlation PARAMETERS.

\begin{tabular}{l|r}
\hline Parameter & Value \\
\hline FFT and IFFT size & $N_{f f t}=128$ \\
Data rate & $320 \mathrm{Mbps}$ \\
Convolutional encoder's rate & $1 / 2$ \\
Convolutional encoder's constraint length & $K=7$ \\
Convolutional decoder & Viterbi \\
Decoding mode & Hard \\
Number of transmitted & 1200 \\
MB-OFDM symbols & QPSK \& DCM \\
Modulation & CM1, $2,3 \& 4$ \\
IEEE Channel model & $N_{D}=100$ \\
Number of data subcarriers & $N_{P}=12$ \\
Number of pilot subcarriers & $N_{G}=10$ \\
Number of guard subcarriers & $N_{T}=122$ \\
Total number of subcarriers used & $N_{Z P S}=37$ \\
Number of samples in ZPS & $N_{S Y M}=165$ \\
Total number of samples/symbol & 100 \\
Number of channel realizations & \\
\hline
\end{tabular}

3) A better error performance can be achieved, compared to the case of the conventional codes, without increase of the total transmission power, because all zero elements carrying no information in the conventional code are now replaced with information-carrying symbols, thus providing a bigger diversity for the transmitted signals.

For a more detailed explanation, let us consider the example of three systems, including a) the conventional MB-OFDM (without STFCs), b) the STFC MB-OFDM with the code $\mathbf{S}_{\mathbf{8} \mathbf{a}}$, and c) the STFC MB-OFDM with the code $\mathbf{S}_{\mathbf{8 b}}$, in the case of QPSK modulation. Clearly, if the modulated symbols $s_{t, m, k}$ within the vector $\overline{\mathbf{s}}_{t, m}$ are drawn from the power-1 constellation in the system (a), power- $1 / 4$ constellation in the system (b), and power-1/8 constellation in the last system, then the average powers transmitted from all $\mathrm{Tx}$ antennas in these three cases are the same at any time. The SCEs for these cases are $1,1 / 4$, and $1 / 8$, respectively. It will be shown later in the simulation section of this paper that, even with $\mathrm{SCE}=$ $1 / 8$, the STFC $\mathbf{S}_{\mathbf{8 b}}$ still provides better error performance than $\mathbf{S}_{\mathbf{8 a}}$ with $\mathrm{SCE}=1 / 4$, and much better error performance than the conventional MB-OFDM system with $\mathrm{SCE}=1$.

\section{Simulation Results}

To examine the performance of the advanced STFC, we ran several Monte-Carlo simulations for the conventional MBOFDM without STFCs, the Alamouti code $\mathbf{S}_{2}$, the conventional order-8 STFC $\mathbf{S}_{8 a}$ in (15), and the advanced STFC (16) $\mathbf{S}_{8 b}$ in (16). Each run of simulations was carried out with 1200 MB-OFDM symbols. As suggested in [13], 100 channel realizations of each IEEE 802.15.3a channel model (CM 1, 2, 3 and 4) were considered for the transmission of each MBOFDM symbol. In simulations, $S N R$ is defined to be the signal-to-noise ratio $(\mathrm{dB})$ per sample in a MB-OFDM symbol (consisting of 165 samples), at each $\mathrm{Rx}$ antenna. It means that, at a certain Rx antenna, $S N R$ is the subtraction between the total power $(\mathrm{dB})$ of the received signal corresponding to the sample of interest and the power of noise $(\mathrm{dB})$ at that $\mathrm{Rx}$ antenna. To fairly compare the error performance of MBOFDM systems with and without STFCs, the average power of the signal-constellation points in the STFC MB-OFDM system is scaled down by a factor of $1 / 2$ for the Alamouti code $\mathbf{S}_{2}, 1 / 4$ for the code $\mathbf{S}_{8 a}$, and $1 / 8$ for the code $\mathbf{S}_{8 b}$. 
In other words, SCEs for $\mathbf{S}_{2}, S_{8 a}$, and $S_{8 b}$ are 1/2, 1/4, and $1 / 8$, respectively. Thereby, the average transmitting power from all Tx antennas at a certain time is kept the same for four cases: conventional MB-OFDM without STFCs, Alamouti MB-OFDM, and the two order- 8 STFC MB-OFDM systems. Both modulation schemes QPSK and DCM are simulated. The simulation parameters are listed in Table II.

Fig. 2 shows that the implementation of an order-8 STFC in the case of QPSK modulation/demodulation and $1 \mathrm{Rx}$ antenna can advance the error performance by at least $6.5 \mathrm{~dB}$ at $\mathrm{BER}=$ $10^{-4}$, compared to the order-2 (Alamouti) STFC MB-OFDM system. Fig. 3 shows that a gain of at least $5 \mathrm{~dB}$ at $\mathrm{BER}=10^{-4}$ can be achieved, compared to the Alamouti STFC MB-OFDM, when the order- 8 STFC is used in the case of DCM modulation and $2 \mathrm{Rx}$ antennas. Thus, these two figures illustrate that the application of order- 8 STFCs can provide much better error performance than the order-2 STFCs, thanks to the higher diversity order, with the cost of half data rate, compared to the full-rate systems (the conventional MB-OFDM system without STFCs, and the Alamouti STFC MB-OFDM system).

Figs. 4 and 5 compare the performance of the conventional MB-OFDM without STFCs and that of the STFC MB-OFDM using $S_{8 a}$ and $S_{8 b}$ in the case of QPSK modulation, with 1 and 2 Rx antennas, respectively. Similarly, Figs. 6 and 7 compare those systems in the DCM scenario. It is clear from these figures that the advanced code $S_{8 b}$ provides the best error performance, without increasing the total transmission power. The more dispersive the channel is, the better the code $S_{8 b}$ is (over the code $S_{8 a}$ ). In other words, even with SCE $=1 / 8, S_{8 b}$ can still provide better error performance than $S_{8 a}$ with $\mathrm{SCE}=1 / 4$. Equivalently, if the restriction of equal total transmission power is released in order that the same average power per QPSK or DCM symbol (the same SCE the signal constellation energy need not to be scaled down) is transmitted, $S_{8 b}$ can provide much better error performance than $S_{8 a}$.

The reason behind these performance advances is that $S_{8 b}$ comprises no wasteful symbols, unlike $S_{8 a}$. All symbols inside the code matrix $S_{8 b}$ are used to transmit information, thus providing more time, space, frequency diversities for the transmitted information.

\section{CONCLUSions}

In this paper, we have analyzed the advantage of using STFCs of up to 8th order, and the feasibility of their deployment. Though the full rate cannot be achieved by higherorder STFCs, a higher diversity order can be obtained. Thus, the rate and diversity order can be compromised, depending on particular applications, to satisfy the expected system performance. We have also pointed out that the code $\mathbf{S}_{\mathbf{8 b}}$ without any zero element, which we have proposed previously for wireless MIMO STBC systems, is an advanced structure, providing better error performance than the conventional code $\mathbf{S}_{\mathbf{8 a}}$ with various zeros, without any increase of the total transmission power.

\section{ACKNOWLEDGMENT}

L. C. Tran would like to thank the Alexander von Humboldt $(\mathrm{AvH})$ Foundation, Germany, for its support of this work in form of a postdoctoral research fellowship.

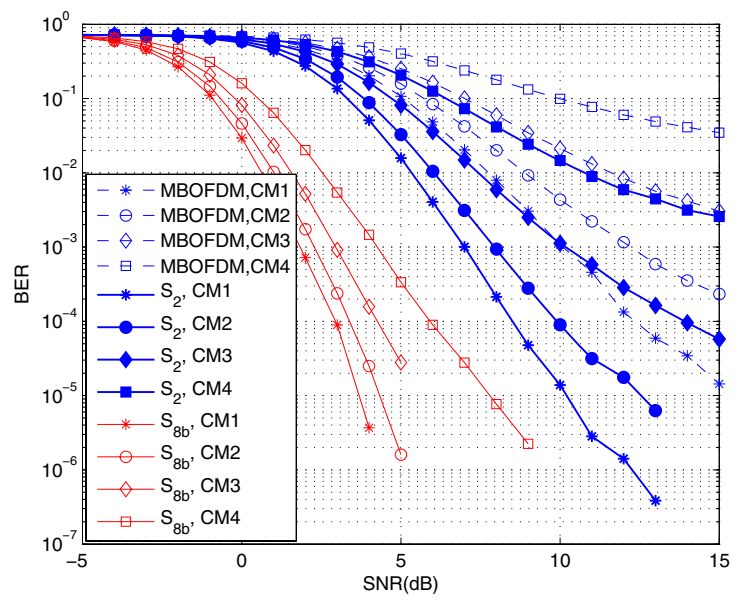

Fig. 2. Comparison between $\mathbf{S}_{\mathbf{2}}$ and $\mathbf{S}_{\mathbf{8 b}}$ with $1 \mathrm{Rx}$ antenna and QPSK modulation/demodulation.

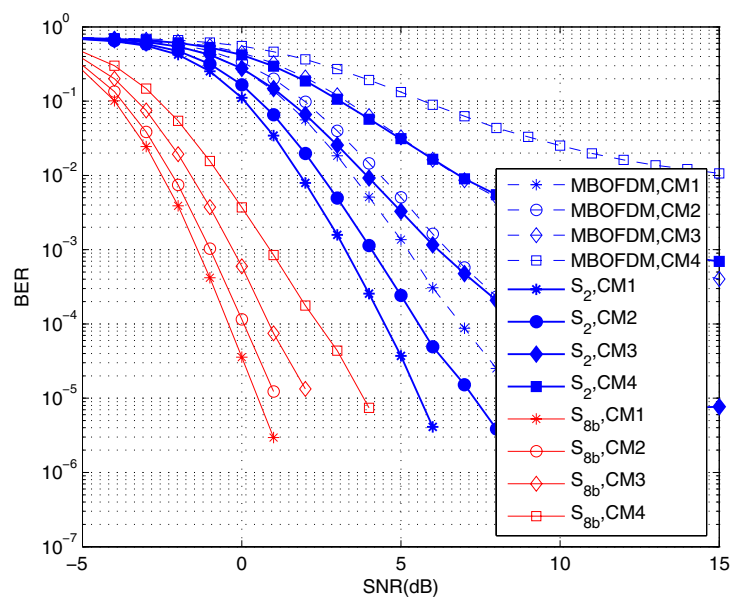

Fig. 3. Comparison between $\mathbf{S}_{\mathbf{2}}$ and $\mathbf{S}_{\mathbf{8 b}}$ with $2 \mathbf{R x}$ antennas and DCM modulation/demodulation.

\section{REFERENCES}

[1] A. Batra et al., "Multiband OFDM physical layer specification," WiMedia Alliance, Release 1.1, July 2005.

[2] I. E. Telatar, "Capacity of multi-antenna Gaussian channels," European Trans. Telecom., vol. 10, no. 6, pp. 585-595, 1999.

[3] G. J. Foschini and M. J. Gans, On limits of wireless communications in a fading environment when using multiple antennas, vol. 6 of 3, Wireless Personal Communications, Printed in the Netherlands, Mar. 1998.

[4] S. M. Alamouti, "A simple transmit diversity technique for wireless communications," IEEE J. Select. Areas Commun., vol. 16, no. 8, pp. $1451-1458$, Oct. 1998.

[5] V. Tarokh, N. Seshadri, and A. R. Calderbank, "Space-time codes for high data wireless communications: performance criterion and code construction," IEEE Trans. Inform. Theory, vol. 44, no. 2, pp. $744-$ 765, Mar. 1998.

[6] V. Tarokh, H. Jafarkhani, and A. R. Calderbank, "Space-time blocks codes from orthogonal designs," IEEE Trans. Inform. Theory, vol. 45, no. 5 , pp. $1456-1467$, July 1999 .

[7] L. C. Tran, T. A. Wysocki, A. Mertins, and J. Seberry, Complex Or- 


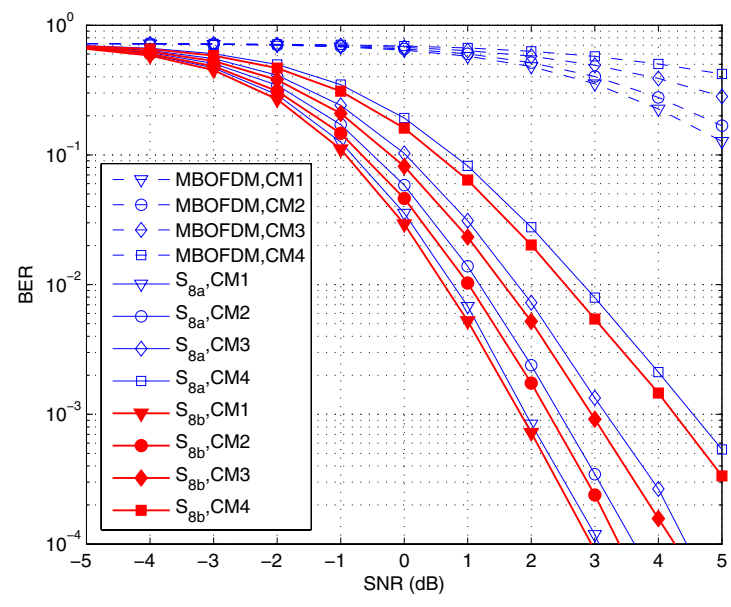

Fig. 4. Comparison between $\mathbf{S}_{\mathbf{8}}$ and $\mathbf{S}_{\mathbf{8 b}}$ with $1 \mathrm{Rx}$ antenna and QPSK modulation/demodulation.

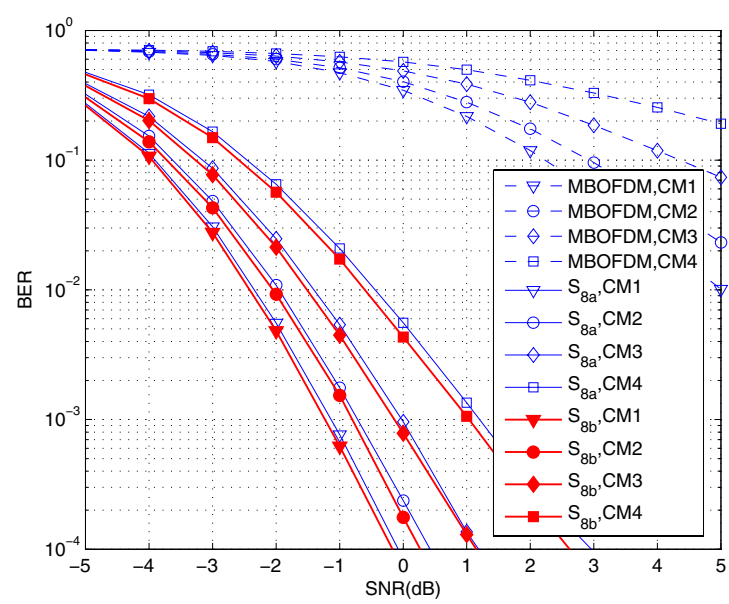

Fig. 5. Comparison between $\mathbf{S}_{\mathbf{8 a}}$ and $\mathbf{S}_{\mathbf{8 b}}$ with $2 \mathbf{R x}$ antennas and QPSK modulation/demodulation.

thogonal Space-Time Processing in Wireless Communications, Springer, New York, USA, 2006.

[8] X.-B. Liang, "Orthogonal designs with maximal rates," IEEE Trans. Inform. Theory, vol. 49, no. 10, pp. 2468-2503, Oct. 2003.

[9] T.-H. Tan and K.-C. Lin, "Performance of space-time block coded MBOFDM UWB systems," Proc. 4th Annual Communication Networks and Services Research Conference (CNSR'06), pp. 323 - 327, May 2006.

[10] W.P. Siriwongpairat, W. Su, M. Olfat, and K.J.R. Liu, "MultibandOFDM MIMO coding framework for UWB communication systems," IEEE Trans. Sign. Process., vol. 54, no. 1, pp. 214 - 224, Jan. 2006.

[11] J. Hou and M. H. Lee, "High rate ultra wideband space time coded OFDM," Proc. 58th IEEE Veh. Technol. Conf. VTC 2003 - Fall, vol. 4, pp. 2449-2451, Oct. 2003.

[12] J. Wang, G. Zhu, and J. Jin, "Optimal power allocation for spacetime coded OFDM UWB systems," Proc. IEEE Int. Conf. Wireles. Communications, Networking and Mobile Computing WCNM.2005, vol. 1 , pp. $189-192$, Sept. 2005 .

[13] J. Foerster et. al., "Channel modelling sub-committee report final," IEEE P802.15 Working Group for Wireless Personal Area Networks (WPANs) IEEE P802.15-02/490r1-SG3a, Oct. 2005.

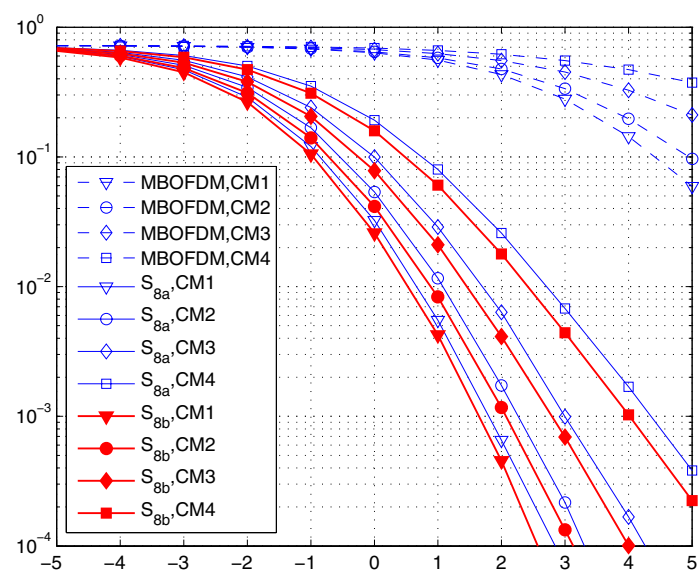

Fig. 6. Comparison between $\mathbf{S}_{\mathbf{8 a}}$ and $\mathbf{S}_{\mathbf{8 b}}$ with $1 \mathrm{Rx}$ antenna and DCM modulation/demodulation.

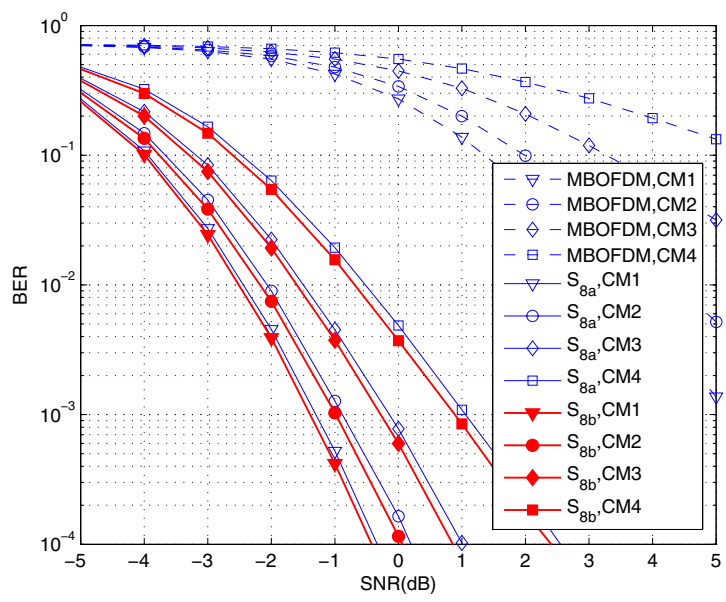

Fig. 7. Comparison between $\mathbf{S}_{\mathbf{8 a}}$ and $\mathbf{S}_{\mathbf{8 b}}$ with $2 \mathrm{Rx}$ antennas and DCM modulation/demodulation.

[14] L. C. Tran and A. Mertins, "Space-time-frequency code implementation in MB-OFDM UWB communications: design criteria and performance,' Accepted for revision in IEEE Trans. Wireless Commun., June 2007.

[15] Y. Viniotis, Probability and random processes for electrical engineers, McGraw-Hill, Printed in Singapore, 1998.

[16] L. C. Tran, T. A. Wysocki, J. Seberry, A. Mertins, and S. A. Spence, "Generalized Williamson and Wallis-Whiteman constructions for improved square order-8 CO STBCs," Proc. 16th IEEE Int. Symp. Personal Indoor and Mobile Radio Communications PIMRC'05, vol. 2, pp. 1155 -1159 , Sept. 2005.

[17] L. C. Tran, J. Seberry, B. J. Wysocki, T. A. Wysocki, T. Xia, and Y. Zhao, "Two new complex orthogonal space-time codes for 8 transmit antennas," IEE Electronics Lett., vol. 40, no. 1, pp. 55-56, Jan. 2004.

[18] V. Tarokh, H. Jafarkhani, and A. R. Calderbank, "Space-time block coding for wireless communications: performance results," IEEE $J$. Select. Areas Commun , vol. 17, no. 3, pp. 451 - 460, Mar. 1999.

[19] O. Tirkkonen and A. Hottinen, "Square-matrix embeddable space-time blocks codes for complex signal constellations," IEEE Trans. Inform. Theory, vol. 48, no. 2, pp. 384 - 395, Feb. 2002. 